\title{
3D Tomography and Image Processing Using Fast Neutrons Final Report
}

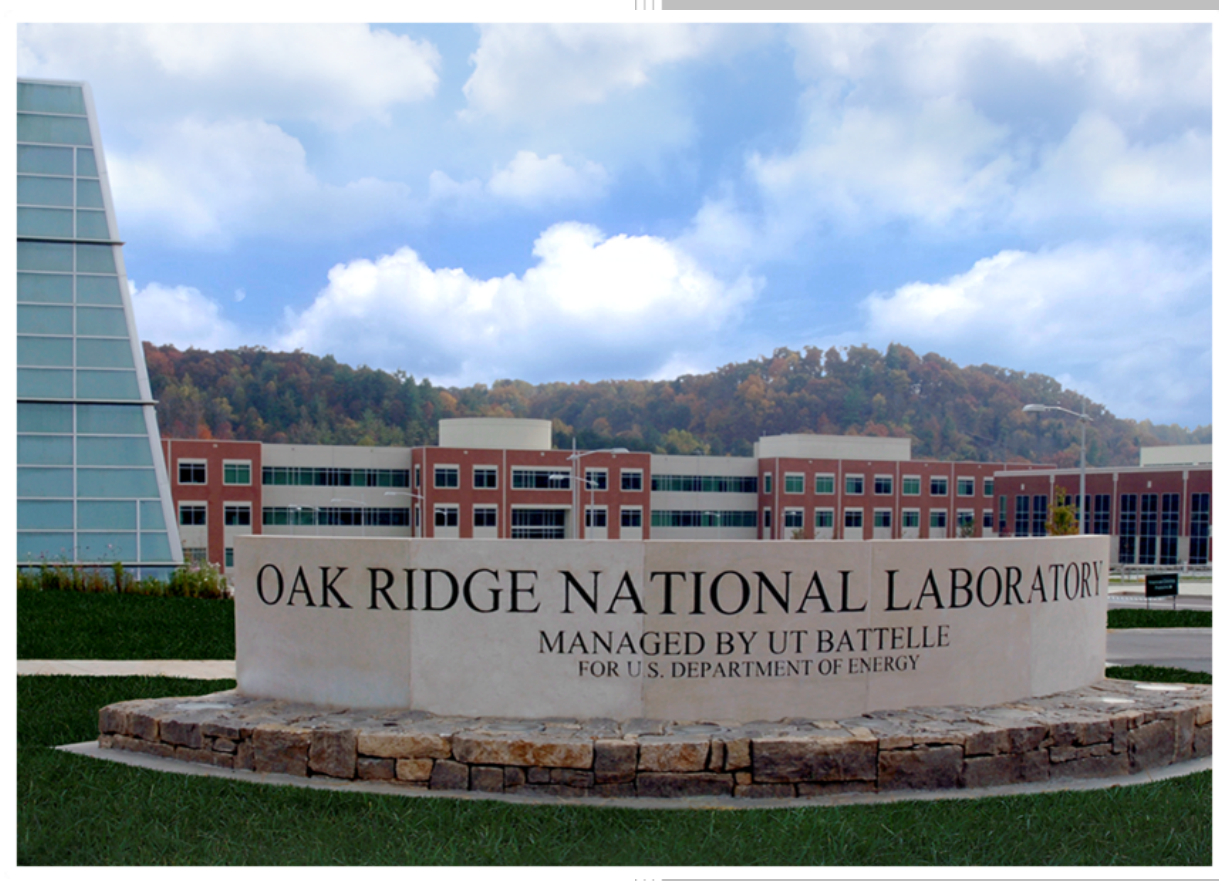

\section{Approved for public release.} Distribution is unlimited.
Paul A. Hausladen Matthew A. Blackston Deniz Aykac John Sparger Jens Gregor Andrew J. Gilbert Richard S. Wittman Erin A. Miller

January 2020 


\section{DOCUMENT AVAILABILITY}

Reports produced after January 1, 1996, are generally available free via US Department of Energy (DOE) SciTech Connect.

Website www.osti.gov

Reports produced before January 1, 1996, may be purchased by members of the public from the following source:

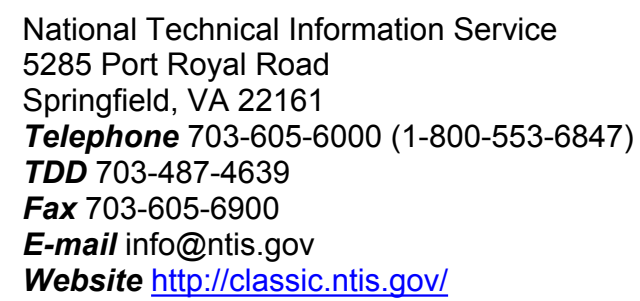

Reports are available to DOE employees, DOE contractors, Energy Technology Data Exchange representatives, and International Nuclear Information System representatives from the following source:

Office of Scientific and Technical Information

PO Box 62

Oak Ridge, TN 37831

Telephone 865-576-8401

Fax 865-576-5728

E-mail reports@osti.gov

Website http://www.osti.gov/contact.html

This report was prepared as an account of work sponsored by an agency of the United States Government. Neither the United States Government nor any agency thereof, nor any of their employees, makes any warranty, express or implied, or assumes any legal liability or responsibility for the accuracy, completeness, or usefulness of any information, apparatus, product, or process disclosed, or represents that its use would not infringe privately owned rights. Reference herein to any specific commercial product, process, or service by trade name, trademark, manufacturer, or otherwise, does not necessarily constitute or imply its endorsement, recommendation, or favoring by the United States Government or any agency thereof. The views and opinions of authors expressed herein do not necessarily state or reflect those of the United States Government or any agency thereof. 
Isotope and Fuel Cycle Technology Division

\title{
3D TOMOGRAPHY AND IMAGE PROCESSING USING FAST NEUTRONS FINAL
} REPORT

\author{
Paul A. Hausladen \\ Matthew A. Blackston \\ Deniz Aykac \\ John Sparger \\ Jens Gregor* \\ Andrew J. Gilbert ${ }^{\dagger}$ \\ Richard S. Wittman \\ Erin A. Miller
}

*University of Tennessee, Knoxville

†Pacific Northwest National Laboratory

January 2020

Prepared by

OAK RIDGE NATIONAL LABORATORY

Oak Ridge, TN 37831-6283

managed by

UT-BATTELLE, LLC

for the

US DEPARTMENT OF ENERGY

under contract DE-AC05-00OR22725 



\section{CONTENTS}

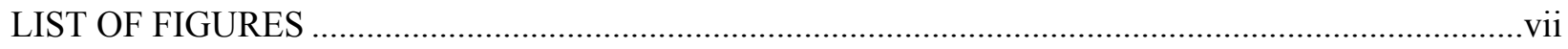

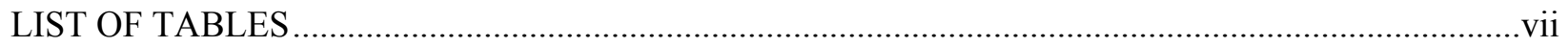

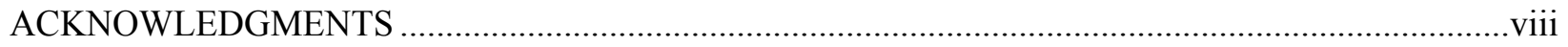

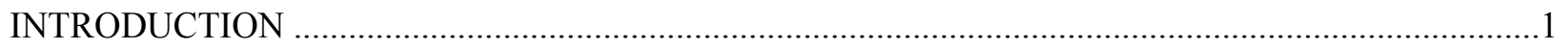

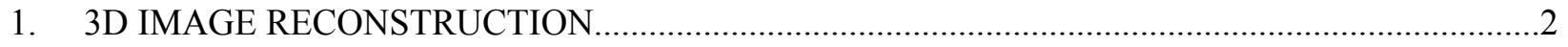

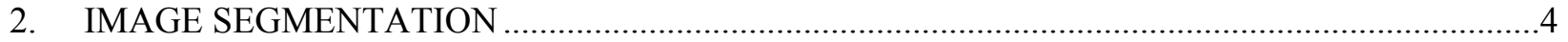

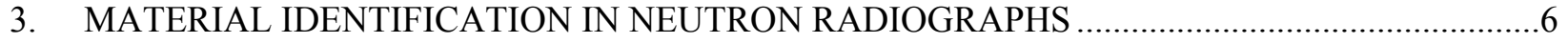

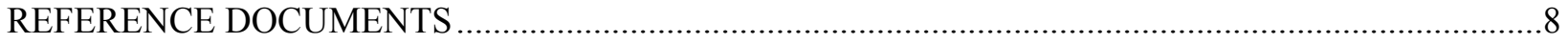





\section{LIST OF FIGURES}

Figure 1. Example 3D reconstruction of target consisting of a DU annulus, an imaging detector, and a water bottle inside a steel tee pipe.

Figure 2. Results of image segmentation using both transmission and induced-reaction reconstruction data for the target consisting of a DU annulus, an imaging detector, and a water bottle inside a steel tee pipe.

Figure 3. (Left) A schematic of the MCNP modeled API system with the three-annulus object in place.

Figure 4. (Left) A simulated radiograph of the three-annuli object using a DT neutron source.

Figure 5. A comparison of the MCNP output from a simple model to that expected from a simple analytical model.

\section{LIST OF TABLES}

Table 1. Root mean square error (RMSE) of material area densities estimated from the algorithm due to variations in radiography system response parameters of interest. 



\section{ACKNOWLEDGMENTS}

This work is supported by the US Department of Energy, Office of Defense Nuclear Nonproliferation R\&D (NA-22) in the National Nuclear Security Administration. 



\section{INTRODUCTION}

This document is the final report for project OR16-ML-3DTomography-PD3Jb, "3D Tomography and Image Processing Using Fast Neutrons.” This project was motivated by the availability of threedimensional (3D) tomographic neutron transmission and induced-reaction data following the development of associated-particle imaging (API) systems with two-dimensional (2D) or "area" neutron detectors such as the Advanced Portable Neutron Imaging System, which is capable of simultaneous measurement of transmission and induced-reaction neutrons.

The API method uses fast ( $14 \mathrm{MeV})$ neutrons produced via the $d+t \rightarrow \alpha+n$ reaction in a D-T neutron generator where detection of the time and position of the alpha particle determines the time and direction of the associated neutron. Use of this coincidence technique enables pencil-beam contrast over a wide cone beam without the need for physical collimators. Use of coincidence methods also enables inducedreaction imaging, where certain neutron interactions such as elastic hydrogen scattering or induced fission can be identified via the arrival times, positions, and number of detected neutrons and associated with the initial neutron direction. Most neutrons that traverse an object thicker than a few mean free paths interact one or more times, so it is desirable to extract information from these neutrons that would otherwise solely be a source of noise.

The use of fast neutrons is preferred for applications where it is desirable to penetrate metal and have contrast for light materials. The combination of fast neutrons and coincidence methods enables portable, low dose imaging that can be brought to an object under inspection and used without a specialized facility. Because of the ability to bring the imager to a facility, potential applications for API neutron imaging include performing nuclear materials accounting, adjudicating incomplete records, assessing safety, performing quality assurance assessments, or evaluating unknown items. To use API for these applications, performing and analyzing tomographic measurements in practical amounts of time is necessary. As a result, the authors have spent the last decade building this capability.

The first step toward developing the desired tomographic 3D imaging capability, which was completed during previous efforts, was the development of fast neutron imaging systems with $2 \mathrm{D}$ detector panels. This accomplishment represented a substantial capability improvement over concurrent and earlier imaging systems having one-dimensional or "line" neutron transmission detectors such as the Nuclear Materials Identification System. Instead of needing to scan the system to build up a single 2D image, the same measurement time could be used to accumulate several projections to perform tomographic imaging. Moreover, systems that have a 2D detector are sufficiently efficient to measure induced reactions in practical measurement times.

The second step toward developing the desired tomographic 3D imaging capability is the subject of the present work - the development of image reconstruction and analysis software capable of digesting the 3D imaging data. In particular, image reconstruction software was desired that was capable of using input data from a wide cone beam, stitching together tomographic measurements at multiple scan heights (although not spiral scan), and calculating the system response for transmission, hydrogen elastic scattering, and induced neutron imaging using a common system geometry. In addition, analysis software that could extract information from the images, such as shapes or materials, was desired. As a result, this project had two overall goals. The first of these goals was to extend associated-particle fast neutron transmission and induced-reaction tomographic imaging algorithms to three dimensions using modern, parallel, iterative reconstruction code. The second goal was to automatically segment the resultant tomographic images into constituent parts and then extract information about the parts, such as the class of shape and potentially parameters that describe it. In the third year of the project, the project was expanded to include an effort to extend a previously developed material identification algorithm for dual- 
energy x-ray radiographs to use transmission fast neutron radiographs from API systems. This third effort was conducted by researchers from Pacific Northwest National Laboratory (PNNL).

The purpose of this report is to provide an executive summary of project accomplishments and provide a list deliverable reports that detail its constituent efforts. For detailed information about each effort, the reader is directed to the referenced deliverable reports.

\section{3D IMAGE RECONSTRUCTION}

At the outset of this project, the iterative reconstruction codes used for transmission and induced-reaction imaging for API systems consisted of prototype scripts implemented in the Matlab software environment that were only capable of producing single 2D tomographic slices through target objects. Although these scripts were sufficient to demonstrate proof-of-concept for induced-reaction imaging, not only were they incapable of 3D reconstruction, but their slow execution speed also effectively precluded their use in performing 3D reconstruction if appropriately modified. In the present work, the Matlab scripts were replaced by fully parallelized code written in the $\mathrm{C}$ programming language with significant improvements in reconstruction speed.

In addition to making full use of the large-area coverage of these imagers, the 3D reconstruction codes are also more correct. In particular, for induced-reaction reconstruction, the system response model uses the calculated transmission to estimate the probability of transporting a neutron from the source to the interaction point and from the interaction point to each detector. Even for a 2D reconstruction, the latter portion of this task is inherently $3 \mathrm{D}$ because it requires transporting neutrons out of the plane where transmission was measured to reach detectors. To perform reconstruction, the original scripts assumed a cylindrical object in its out-of-plane extent, an assumption that may be false. Estimating the system response using the true $3 \mathrm{D}$ geometry is important for accurate reconstructions.

During this project, a reconstruction framework was successfully developed for producing 3D transmission and induced-reaction images. The framework achieves sufficient speed for practical 3D reconstruction from efficient code and parallelized algorithms; approaches favorable solutions using regularization, penalties, or constraints; and weights data by the appropriate errors. The reconstructions are performed in $3 \mathrm{D}$, including the ability to handle multi-height projection data from a scan, with the paths from source-to-voxel and voxel-to-detector accurately represented in a common system geometry for transmission and induced-reaction imaging.

The following list provides highlights of the new code. More detailed descriptions of each item and additional references can be found in Reference [1].

- 3D representation of the system: Accurate reconstruction requires accurate representation of the detector geometry in 3D, including appropriate treatment of gaps between detectors. All imaging modalities rely on modeling lines of response from the source through the target and continuing, either along the same path in the case of transmission or along new paths in the case of induced reactions, to the detectors. In the new code, the source, detector, and object geometries are modeled in 3D, including accurate locations of detector pixels that account for gaps between detectors. This enables accurate calculation of the attenuation of initial and induced-reaction neutrons along their 3D paths through the target object. The reconstruction accepts projection data from multiple scan heights and solves for reconstructed voxel values using data from all the heights simultaneously. The code also provides an automatic correction for misalignment of the target rotation axis from the center of the imager. 
- Consistent uncertainty treatment: At the outset of this project, image reconstruction used the maximum-likelihood, expectation-maximization (MLEM) algorithm. MLEM assumes that measured values are Poisson-distributed around their true value. However, this assumption is not true when the data has been background-subtracted or, in the case of transmission, the logarithm of the counts has been taken. MLEM was replaced with the simultaneous iterative reconstruction technique (SIRT), which is based on a least squares formalism that can handle the non-Poisson uncertainties associated with API data. SIRT was implemented to allow the user to supply variances for the projection data, making the reconstruction a solution to a weighted least squares (WLS) problem.

- Constraints and regularizations: To reconstruct images that exhibit smooth regions with welldefined edges consistent with machined parts, the mathematical imaging model was extended to consist of a WLS minimization problem that is subjected to a total-variation constraint. The user can supply the code with a target maximum total-variation value or allow the code to select a total-variation target value. In addition, the code can be run with or without regularizations that penalize undesirable solutions, using either a minimum norm or finite differences regularization.

- Tunable imager response: Response parameters can be supplied to the code to more accurately account for the realistic response of the imager for induced-reaction reconstructions. The lines of response for induced reactions are correlated to the initial direction as provided by the alpha detector, and the angular uncertainty on this initial direction can be specified as a fixed angular value or provided as a table of values associated with directions pointing to each neutron pixel. The originating location of neutrons in the neutron generator can be specified as a point or as a disk of user-specified diameter. The detector efficiency as a function of neutron energy, important for induced-reaction efficiency calculations, can be specified globally or for each detector.

- Execution speed: The speed of image reconstruction has been increased substantially. The increases in speed originate from several factors. The new code uses multithreaded, compiled (rather than interpreted) code written in the $\mathrm{C}$ programming language. The new code implements technique of ordered subsets to increase convergence speed and reduce the required number of iterations. In addition, the new code uses sparse matrices to avoid performing operations on a large number of zero-valued elements. Comparisons of the compiled 2D version of the new code to the original 2D Matlab script resulted in execution speed increases of more than 2 orders of magnitude.

In addition to validated reconstruction code with the appropriate functionality and execution speed, the project had a number of other notable efforts:

- Joint estimation of transmission and small angle scatter: Initial steps were taken to develop an improved estimation of the number and distribution of neutrons that undergo small angle scattering and are detected at times consistent with transmission. Accurately subtracting these scattered neutrons will improve the quantitative transmission values that are reconstructed, and also provides more accurate information about backgrounds for all induced signatures. The effort demonstrated that the physics of the interaction can be adequately modeled with the addition of a single quantity to each image voxel, the angular variance that characterizes the shape of the elastic scattering cross section. However, additional development is required before this functionality can be incorporated into the reconstruction code.

- 3D image visualization: Scripts were developed to visualize 3D images of transmission reconstructions with overlays of induced-reaction reconstructions using the ImageJ image processing package. An example image is shown in Figure 1. 

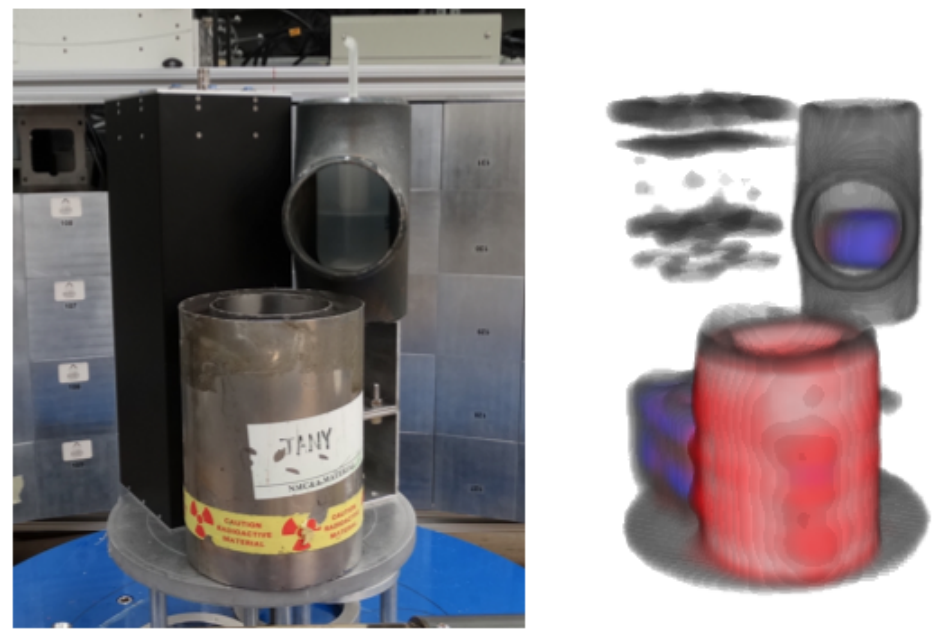

Figure 1. Example 3D reconstruction of a target consisting of a depleted uranium (DU) annulus, an imaging detector, and a water bottle inside a steel tee pipe. (Left) Photograph of the target object and (right) the reconstructed results showing the transmission reconstruction (gray scale) overlaid with induced doubles (red) and hydrogen elastic scatter (blue) reconstructions. Some of the details of the components inside the imaging detector are visible in the transmission image. The induced doubles image correctly highlights the DU annulus, and the hydrogen scatter image correctly highlights the plastic scintillator of the imaging detector (left of the DU annulus) and the water in the water bottle (inside the steel pipe).

\section{IMAGE SEGMENTATION}

Imaging techniques have been developed as high-confidence methods for nondestructively evaluating objects of interest. Because of the excellent contrast with high atomic number-shielding and inducedreaction imaging, API neutron imaging can be used to perform high-confidence confirmation of the presence and configuration of special nuclear materials. This high confidence is achieved at the cost of revealing considerable information that may be undesirable to share with the operator of the equipment. One avenue for benefiting from the high confidence of imaging methods without revealing the imaging data to the operator is to employ automated analysis that can extract meaningful attributes of the special nuclear materials, such as the mass. An essential step of this automated analysis is the segmentation of the image into its constituent parts.

As a result, a key goal of the present work is to infer the boundaries of objects in fast neutron tomographic images via the general method of image segmentation. Development of this capability can be broken down into the following more manageable steps:

1. Operator-guided segmentation of two-dimensional (2D) fast neutron tomographic images

2. Operator-guided segmentation of $3 \mathrm{D}$ fast neutron tomographic images

3. Development of automated segmentation algorithms

4. Extraction of shape parameters from constituent volumes in $3 \mathrm{D}$

The present work implemented operator-guided segmentation of 2D and 3D objects using a multiphase "level set" approach to image segmentation. This project represented the first application of this approach to measured 3D neutron imaging data with mixed success. In the multiphase level set approach, the segmentation problem is formulated as a minimization of the Mumford-Shah energy functional, which is 
derived under the assumptions that the image varies smoothly or slowly and that it varies discontinuously or rapidly across most of the boundaries between the objects. The level set formulation of Vese and Chan [2] provides a framework to calculate the optimal segmentation by minimizing the energy functional. This formulation allows for automatic topology changes such as cusps and corners, operates on a grid, and automatically handles problems associated with gaps and overlaps between regions. This formulation also provides a natural way to include additional information from induced-reaction images as a separate level set, albeit with the added complication that the transmission and induced-reaction images have different resolutions. Figure 2 shows an example segmentation using data from the target shown in Figure 1. A more detailed description of the algorithms that were implemented and additional segmentation results can be found in References [3] and [4].

(a)

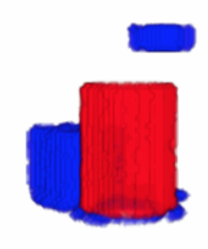

(b)

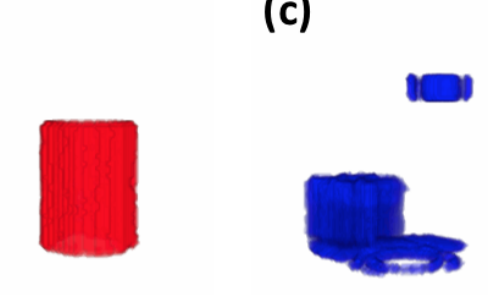

Figure 2. Results of image segmentation using both transmission and induced-reaction reconstruction data for the target consisting of a DU annulus, an imaging detector, and a water bottle inside a steel tee pipe. Segmentation regions shown (a) together and shown separately in (b) and (c). The red region corresponds to the fissionable region, and the blue region corresponds to the hydrogenous region.

The primary challenge for this approach is that it is being applied to relatively noisy, low resolution images. API neutron images are inherently noisy because of the small neutron doses involved in forming the images. Likewise, the large pixels used in the thick transmission detectors limit resolution so that boundaries between materials are often fuzzy. A secondary challenge was that it was being applied to images produced by a code that had not been entirely debugged. As a result, the effective role of the image segmentation portion of the project was sometimes to perform rigorous quantification of images that appeared acceptable to the eye but had flaws due to being produced by code that had undiscovered bugs.

Despite these difficulties, in some cases material regions were successfully segmented using the transmission reconstruction data alone, typically when the separation between relevant volumes was large. In other cases, the transmission images needed to be supplemented with the induced-reaction reconstructions to separate different materials with similar transmission image values. There were still other cases that even the additional information provided by induced-reaction images did not allow regions to be adequately separated. Methods that postprocessed the segmentation results to eliminate edge points attributed to the wrong region were explored and implemented to some success. In addition, an attempt at modifying the energy functional to account for bias and noise in the imaged data was initiated but abandoned because the scale for undesirable variations was similar to the scale of object dimensions.

One issue in the image data that was highlighted during the image segmentation implementation was that the same contiguous material was observed to take on a bimodal distribution of image values that depended on the material that was shielding it. For example, if DU was shielded on one side by steel and on the other by polyethylene, then the reconstructed image values were larger for the polyethyleneshielded DU than the steel-shielded DU. The discrepancy is due to the difference in the distributions of 
neutrons that scatter from each shielding material, and the resulting scatter correction during the transmission analysis estimated the small angle scattering with variable accuracy.

Future development of image segmentation methods for tomographic, fast neutron imaging systems will need to address several issues. The highest priority is the improvement of data analysis and image reconstruction algorithms, especially the development of a more accurate scatter correction for transmission imaging so that more accurate representations of the imaged data can be input to the segmentation routines. Improved ways of handling fuzzy edges and noise in the images should be explored, as should adjustments to the energy functional so that segmentation solutions with stray islands of voxels are properly penalized and larger bulk volumes are preferred. Finally, the treatment of transmission and induced-reaction reconstruction images in a single algorithm, possibly by the inclusion of additional level sets for induced-fission, hydrogen scatter, or a combination of both, should be studied. Overall, the segmentation results showed that the use of the combination of transmission, fission, and hydrogen scatter data to segment 3D images has enormous potential for identifying the location of distinct materials within unknown configurations.

\section{MATERIAL IDENTIFICATION IN NEUTRON RADIOGRAPHS}

Noninvasive interrogation of objects opaque to x-rays makes quantitative measurements difficult for a variety of applications. Fast neutrons present a unique interrogation method because they are more penetrating for typical x-ray opaque materials. The PNNL effort involved applying a material identification inverse algorithm for quantification of materials to simulated neutron API radiographs. This work can be summarized in three main thrusts:

1. A Monte Carlo model of the ORNL API system was generated, including timing information, to gain an intuitive understanding of the system and the various physics observables involved. As a result, the modeling framework agrees well with API data and was used to quickly generate models of interest.

2. The most recent iteration of the material identification algorithm was developed, tested, and debugged. This included completing a sensitivity study on the algorithm response to several variations in the API system response (e.g., scatter removal and spatial resolution). Consequently, the algorithm is performing nominally and ready for testing with more complex objects and observables.

3. Other physics observables from the API system, particularly the gammas that are emitted from an object due to a variety of neutron interactions, were studied. As a result, an analytical model was developed that agrees reasonably well with modeled data.

For the first thrust area, an MCNP modeling framework was developed that models the time-dependent flux response at an image plane for tagged fast neutrons traveling through an object. Though high-fidelity GEANT4 models of the system already exist at ORNL, the PNNL team developed an MCNP simulation framework to gain an intuitive understanding of the system and to be able to quickly generate simulations of interest.

A target model was developed consisting of a cylindrical array of neutron tallies with three nominal annuli with variable material composition. Figure 3 shows a schematic of the simulated system and the timing information that is obtained from the simulation. Good agreement is observed between the simulated data and a scaled version of the measured data (detector efficiency was not modeled). More details on the model can be found in Reference [5]. 

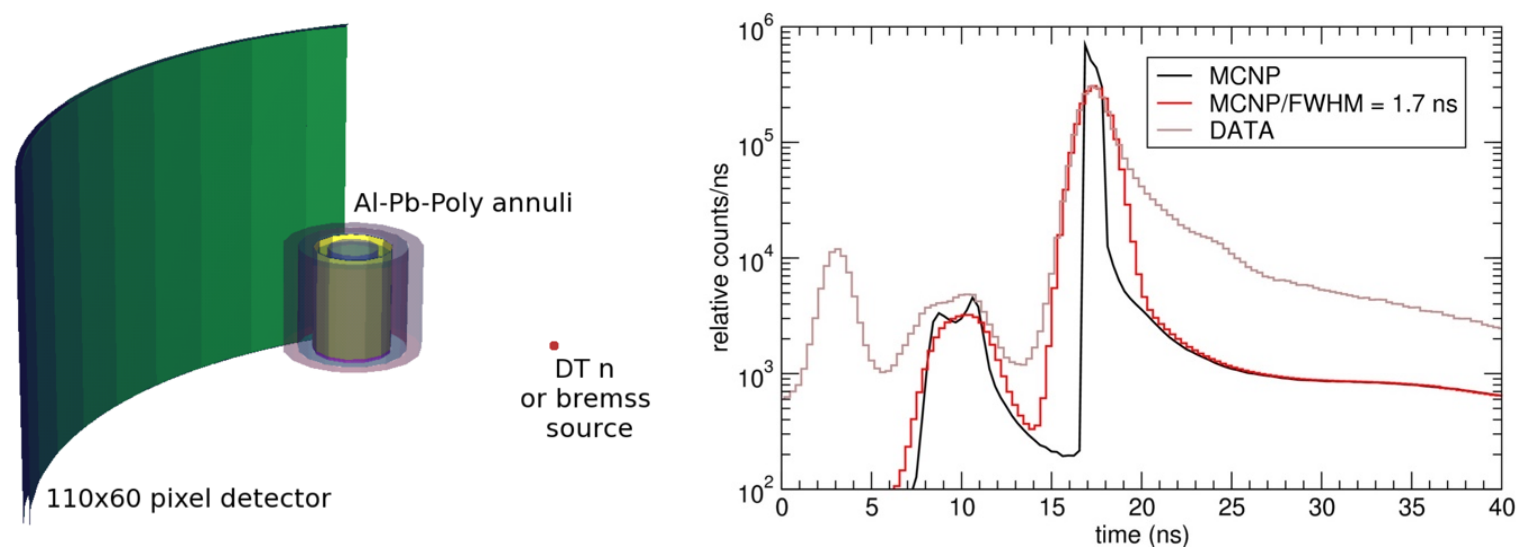

Figure 3. (Left) A schematic of the MCNP modeled API system with the three-annulus object in place. The locations of the neutron tallies are shown in green. (Right) The timing information from the simulation, along with a scaled comparison to measured data of the three-annulus object.

For the algorithm development and testing thrust, a new version of the algorithm was generated for application to API data. The algorithm was constructed to be flexible and allow for a variety of inspected objects and system responses. Figure 4 shows an example of a modeled radiograph as well as the output from the algorithm as a set of estimated materials densities. Here, the three-annuli object is composed of lead, aluminum, and polyethylene, ordered from inner to outer annulus. Simulated radiographs from a DT neutron source, as well as 6 and $9 \mathrm{MVp}$ bremsstrahlung x-ray spectra were input to the algorithm. The noisy results are mostly caused by limited statistics attributable to lead being very opaque to x-rays.
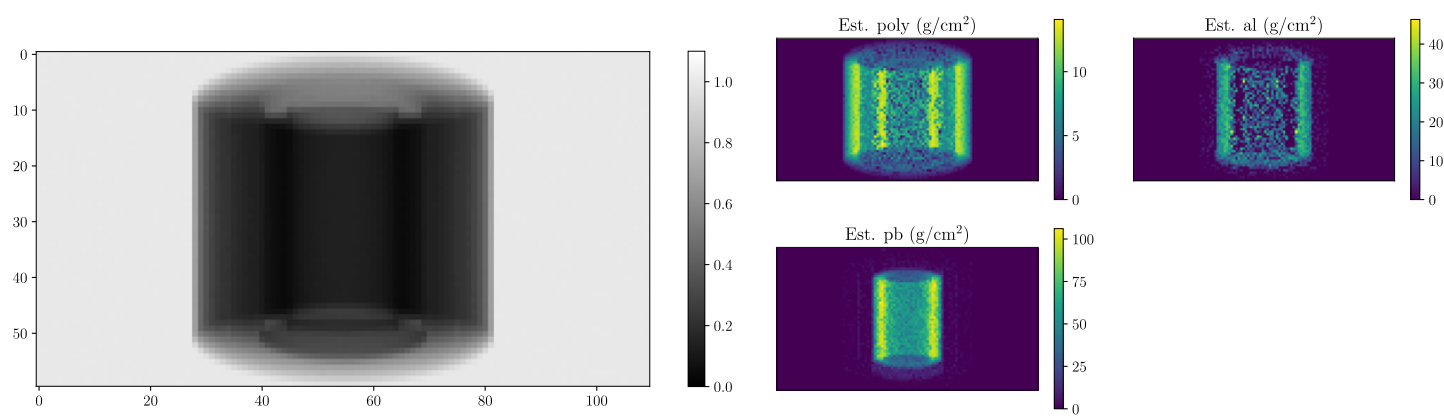

Figure 4. (Left) A simulated radiograph of the three-annuli object using a DT neutron source. (Right) The output from the material identification algorithm. The quality of the reconstruction is limited by the relatively high noise in the simulation.

The algorithm was also tested by completing a sensitivity study to determine how inaccuracies in the physics model supplied to the material identification algorithm affect the algorithm output. A summary of the material errors estimated from the sensitivity study is shown in Table 1 for a few system response parameters of interest. In measuring the errors from the algorithm, the algorithm is most sensitive to the amount of scatter included in the model. More details about the algorithm and sensitivity study can be found in Reference [5]. 
Table 1. Root mean square error (RMSE) of material area densities estimated from the algorithm due to variations in radiography system response parameters of interest.

\begin{tabular}{c|ccc}
\hline & \multicolumn{3}{|c}{ Material RMSE $\left[\mathrm{g} / \mathrm{cm}^{2}\right]$} \\
Parameter & Poly & $\mathrm{Al}$ & $\mathrm{Pb}$ \\
\hline Image noise, $[0.01 \%, 0.1 \%]$ & 0.27 & 1.2 & 0.83 \\
Scatter fraction, $f=0.01$ & 0.806 & 3.491 & 2.198 \\
Image resolution, $\sigma_{P S F}=0.4$ & 0.601 & 2.40 & 1.10
\end{tabular}

The final thrust area was to begin an exploration into the other physics observables from the API system. This involves considering which neutron interactions result in measurements that can be used to identify and quantify material composition of an object. The OpenMC software tool was used to compile neutron cross sections and subsequent gamma emissions that are of interest for material identification. An analytical model of a point-like object was developed that can determine the spectral gamma emissions from any number of materials. The result of this model was compared to an MCNP model with a similar geometry (Figure 5). The agreement is reasonable, and some discrepancies can be explained.

Nevertheless, this study has begun to show how the uncertainties in the underlying nuclear data may limit the ability to determine material composition information from the gamma emission data. More details on the gamma emission study can be found in the Reference [6].

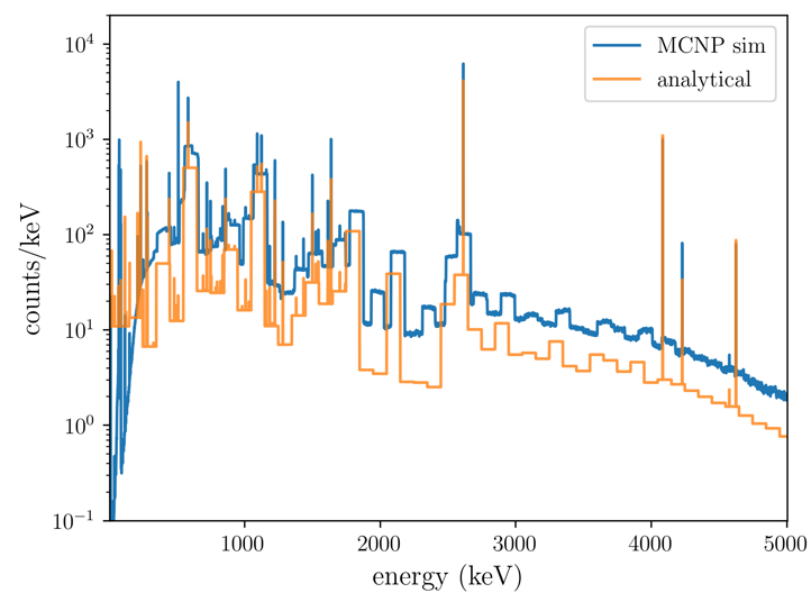

Figure 5. A comparison of the MCNP output from a simple model to that expected from a simple analytical model.

\section{REFERENCE DOCUMENTS}

[1] P. A. Hausladen, M. A. Blackston, and J. Gregor, Progress Update on Iterative Reconstruction of Neutron Tomographic Images, 2019, Report No. ORNL/SPR-2019/1353, Oak Ridge National Laboratory, Oak Ridge, TN, September 2019.

[2] L. A. Vese and T. F. Chan, "A multiphase Leve set framework for image segmentation using the Mumford and Shah model," International Journal of Computer Vision 50(3), 271-293, 2002. 
[3] P. A. Hausladen, D. Aykac, M. A. Blackston, and J. Sparger, Progress Update on Segmentation of Neutron Tomographic Images, 2017, Report no. ORNL/LTR-2017/285, Oak Ridge National Laboratory, Oak Ridge, TN, 2017.

[4] M. A. Blackston, D. Aykac, P. A. Hausladen, Progress Update on Segmentation of Neutron Tomographic Images, 2018, Report no. ORNL/SPR-2018/925, Oak Ridge National Laboratory, Oak Ridge, TN, September 2018.

[5] A. J. Gilbert, R. S. Wittman, E. A. Miller, Material ID with Neutron and X-ray Radiography, Report no. 28813, Pacific Northwest National Laboratory, Richland, WA, 2019.

[6] A. J. Gilbert, R. S. Wittman, E. A. Miller, Prompt Gamma Emission for Material Identification, Report no. 29512, Pacific Northwest National Laboratory, Richland, WA, 2019. 
